\title{
The influence of injecting an epidural contrast agent into the sacral canal on the fluoroscopic visibility of bony landmarks for sacroiliac screw fixation: a feasibility study
}

\author{
Thomas Mendel, MD, PhD, ${ }^{1,2}$ Florian Radetzki, MD, ${ }^{3}$ Stefan Schwan, $\mathrm{PhD},{ }^{4,5}$ \\ Gunther Olaf Hofmann, MD, PhD, ${ }^{1,2}$ and Felix Goehre, MD ${ }^{4,6,7}$
}

\begin{abstract}
1Department of Trauma Surgery, Friedrich-Schiller-University, Jena; Departments of ${ }^{2}$ Trauma Surgery and ${ }^{6}$ Neurosurgery, BG-Kliniken Bergmannstrost; ${ }^{3}$ Department of Orthopedic Surgery, Martin-Luther-University Halle-Wittenberg; ${ }^{5}$ Fraunhofer Institute for Mechanics of Materials, Halle; ${ }^{4}$ Translational Centre of Regenerative Medicine, University of Leipzig, Germany; and ${ }^{7}$ Department of Neurosurgery, Helsinki University Central Hospital, Helsinki, Finland
\end{abstract}

\begin{abstract}
OBJECT In sacroiliac screw fixation of unstable pelvic injuries in geriatric patients, poor bone quality often obscures important bony landmarks in fluoroscopic images. The authors analyzed the feasibility of injecting a transhiatal contrast agent $(\mathrm{CA})$ into the sacral canal to improve fluoroscopic visualization in the sacral epidural space.

METHODS Eight fresh cadaveric whole-body specimens from human donors whose mean age at the time of death was 78 years (range 69-87 years) were used. First, to identify bony landmarks without CA enhancement, the authors acquired fluoroscopy images of the native sacral canal, using lateral, inlet, and outlet projections. Through puncture of the sacral hiatus, 8-10 $\mathrm{ml}$ of CA was injected into the epidural space. Fluoroscopy images were then acquired in the standard pelvic views to identify the bony landmarks. To assess the effect of the CA enhancement, visibility of the landmarks was assessed before and after CA injection. Each identified landmark was scored as 1, and summative landmark scores of up to 10 were determined for each specimen.
\end{abstract}

RESULTS The cadaveric specimens were representative of bone structures in the geriatric population. In all specimens, epidural CA injection enhanced the fluoroscopic visualization of the sacral canal and of the S-1 foramina. The enhancement increased the total bony landmark score from 5.9 (range 4-8) without CA injection to 8.1 (range 6-10) after CA injection. Considering only intrasacral landmarks, the score was increased from 1.5 to 3.

CONCLUSIONS Injection of a transhiatal epidural CA improves fluoroscopic imaging of the sacral canal and of the neural foramina. Hence, this technique could be applied to help the surgeon identify anatomical landmarks during sacroiliac screw fixation in geriatric patients.

http://thejns.org/doi/abs/10.3171/2014.10.SPINE14160

KEY WORDS sacroiliac screw fixation; epidural contrast agent injection; sacral hiatus; fluoroscopy; pelvic injury in the elderly; sacral

$\mathrm{P}$ ERCUTANEOUS sacroiliac (SI) screw fixation is an established technique for stabilizing selected types of fractures in unstable pelvic injuries. It is the only minimally invasive method for treating instabilities of the posterior pelvic ring. Indications for the use of percutaneous SI screw fixation include SI joint dislocations, transiliac and transsacral fracture dislocations, and sacral fractures. However, for a strict percutaneous procedure, fractures need to be nondisplaced or to be reducible in a closed manner.
Because of the low morbidity rate associated with the use of percutaneous SI screw fixation, it is increasingly applied in older patients (that is, in those 65 years or older) to treat fatigue fractures or osteoporotic fractures after low-energy impacts. These types of injuries will become increasingly important because of demographic changes in Western countries. During the last 2 years, 165 patients were hospitalized at our institution for treatment of pelvic fractures, 71 (43\%) of whom were 65 years or older.

However, the complex anatomy and variability in the

ABBREVIATIONS $\mathrm{CA}=$ contrast agent; $\mathrm{SI}=$ sacroiliac

SUBMITTED February 11, 2014. ACCEPTED October 23, 2014

INCLUDE WHEN CITING Published online November 28, 2014; DOI: 10.3171/2014.10.SPINE14160.

DISCLOSURE The authors report no conflict of interest concerning the materials or methods used in this study or the findings specified in this paper. 
shape of the sacrum in different populations make treatment with percutaneous SI screw fixation technically challenging. Limited fluoroscopic visualization of bone structures places large demands on the surgeon's visual thinking. ${ }^{7}$ In addition, the low mineral density of the bone in older patients decreases visibility of bony landmarks during C-arm fluoroscopy. The bony sacral canal and the S-1 neural foramina are both important structures for helping the surgeon achieve sufficient visual orientation.

The interconnections between the sacral canal and neural foramina lead to the complex 3D structure of the sacral cavity (Fig. 1), which consists of an intra- and extradural cavity separated by the dura mater. Traditionally, myelography is used to visualize pathological abnormalities in the spinal channel via intradural injection of a contrast agent (CA). This method enables visualization of the structures in the intradural space, which ends anywhere from S-1 up to the S-3 segment levels ${ }^{11}$ and includes the exiting nerve root sheaths just before their entrance into the osseous foramina. Thus, conventional myelography is not helpful for gaining structural information about the intraoperative anatomy of the inner sacrum peripheral to the nerve root sheaths of the sacrum.

In contrast, a retrograde transhiatal CA instillation into the fat-filled epidural space of the sacrum may facilitate improved imaging of essential bony structures for SI screw fixation. Currently, this injection technique is widely incorporated by anesthesiologists, for example, for caudal epidural blockade in pain patients..$^{12,16}$ The aim of this study was to examine the utility of using a CA in the sacral epidural space to improve percutaneous SI screw fixation of osteoporotic bone.

\section{Methods}

For this cadaveric study, 8 unfixed whole-body human specimens were used. Specimens were from anonymous donors and obtained from the Martin-Luther-University Halle-Wittenberg. During their lifetime, these donors expressed the desire to provide their body for medical and scientific purposes after their demise. The donated specimens were from 6 women and 2 men, with a mean age of 78 years (range $69-87$ years).

For the epidural injection, a 21-gauge spinal needle was used. With the specimen in the prone position, the sacral

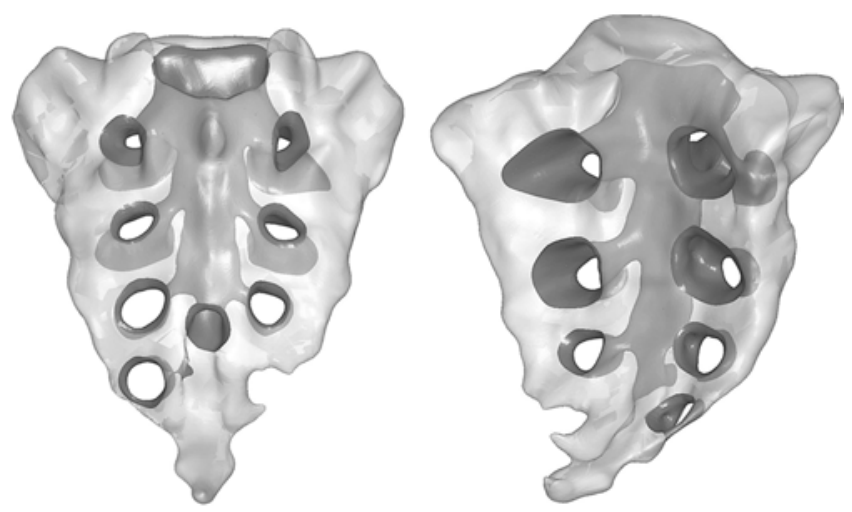

FIG. 1. Computed tomography reconstruction of the sacral channel and neural foramina in posterior and oblique views. hiatus was identified by palpation of the spinous processes and the sacral cornua, which form an isosceles triangle with the posterior superior iliac spines. The injection was performed by puncture through the sacrococcygeal membrane. ${ }^{3,11,14}$ The needle was then further advanced for approximately $1 \mathrm{~cm}$, during which its attitude was monitored with a C-arm. With fluoroscopic monitoring, 8-10 $\mathrm{ml}$ of Ultravist 240 (Bayer Vital GmbH) was gently injected into the caudal epidural space.

For fluoroscopic viewing, a Vision FD flat detector Carm (Ziehm) was used. As is done during surgical procedures for SI screw fixation, standard fluoroscopic C-arm images, namely, strictly lateral and inlet and outlet projections, were acquired for each human specimen before and after injection of the CA into the sacral hiatus. The lateral projection is commonly used for detecting the screw entry point on the iliac surface. With both the inlet and outlet views, the progression of the screw through the sacral alar isthmus can be controlled. However, this view is limited ventrocranially by the sacral ala, caudally by the descending neural foramina, and dorsomedially by the sacral canal. ${ }^{6}$

To ensure accurate screw insertion, the surgeon implements the aforementioned $\mathrm{C}$-arm projections, while recognizing limiting bone structures, to define the correct bone corridor. Table 1 shows the essential bony landmarks that must be found in each fluoroscopic image to permit reliable evaluation of the bone corridors in the first sacral segment. ${ }^{6}$ In fact, the sacral cavity landmarks, such as the sacral canal and the S-1 neural foramina, are often poorly visible or not visible at all. Figure 2 shows the relevant cavity landmarks in a young individual with good bone quality.

For a comparative assessment of the visibility of all essential bony landmarks (Table 1) under fluoroscopy, a simple summative score was devised. One specialist trauma surgeon (T.M.) and one specialist neurosurgeon (F.G.) developed this score while taking into account the surgical demands of daily application of SI screw fixation techniques. For scoring, each of the limiting bony landmarks received a score of 1 if it could be clearly detected in the fluoroscopy image. If the landmark could not be identified, the score was recorded as 0 . This scoring was evaluated for each of the 3 standard C-arm views. Hence, the score could reach a maximum of 4 in the lateral projection and of 3 for each of the inlet and outlet projections, resulting in a total of 10. For the evaluation of the visibility of the sacral cavity landmarks that are directly influenced by the epidural CA injection in the specific projections (that is, the S-1 neural foramina and the sacral canal), the total score value could reach at maximum of 4 (Table 1, Fig. 2). The C-arm evaluation, the contrast injection, and the subsequent image analysis were performed by an experienced, impartial orthopedic surgeon who was neither involved in the score tabulation nor in the later data analysis.

Statistical data analysis was performed using the software Minitab version 16.2.0 (Minitab Ltd). To account for the low number of human specimens, the Fisher exact test and a crosstab calculation were used to test the statistical significance of improved visibility of the individual landmarks after CA injection. A confidence interval of $95 \%$ was used $(p=0.05)$. 
TABLE 1. Summative overall scores and scores including only the landmarks of the sacral cavity

\begin{tabular}{|c|c|c|c|c|c|}
\hline Landmark No.* & Landmark & Score for Lat View & Score for Inlet View & Score for Outlet View & Total Score for All Views \\
\hline 1 & S-1 upper endplate & - & - & 1 & 1 \\
\hline 2 & Sacral ala & - & 1 & 1 & 2 \\
\hline 3 & S-1 neural foramina† & 1 & - & 1 & 2 \\
\hline 4 & Sacral canal† & 1 & 1 & - & 2 \\
\hline 5 & Linea terminalis & 1 & - & - & 1 \\
\hline 6 & S-1 anterior wall & 1 & 1 & - & 2 \\
\hline $1-6$ & All & 4 & 3 & 3 & 10 \\
\hline $3 \& 4$ & Sacral cavities $\dagger$ & 2 & 1 & 1 & 4 \\
\hline
\end{tabular}

$-=$ not assessed in this view.

* The numbering in this column corresponds to that in Fig. 2.

$\dagger$ The visibility of these 2 sacral cavity landmarks was directly influenced by the epidural CA injection, and these landmarks are marked by the asterisks in Fig. 2.
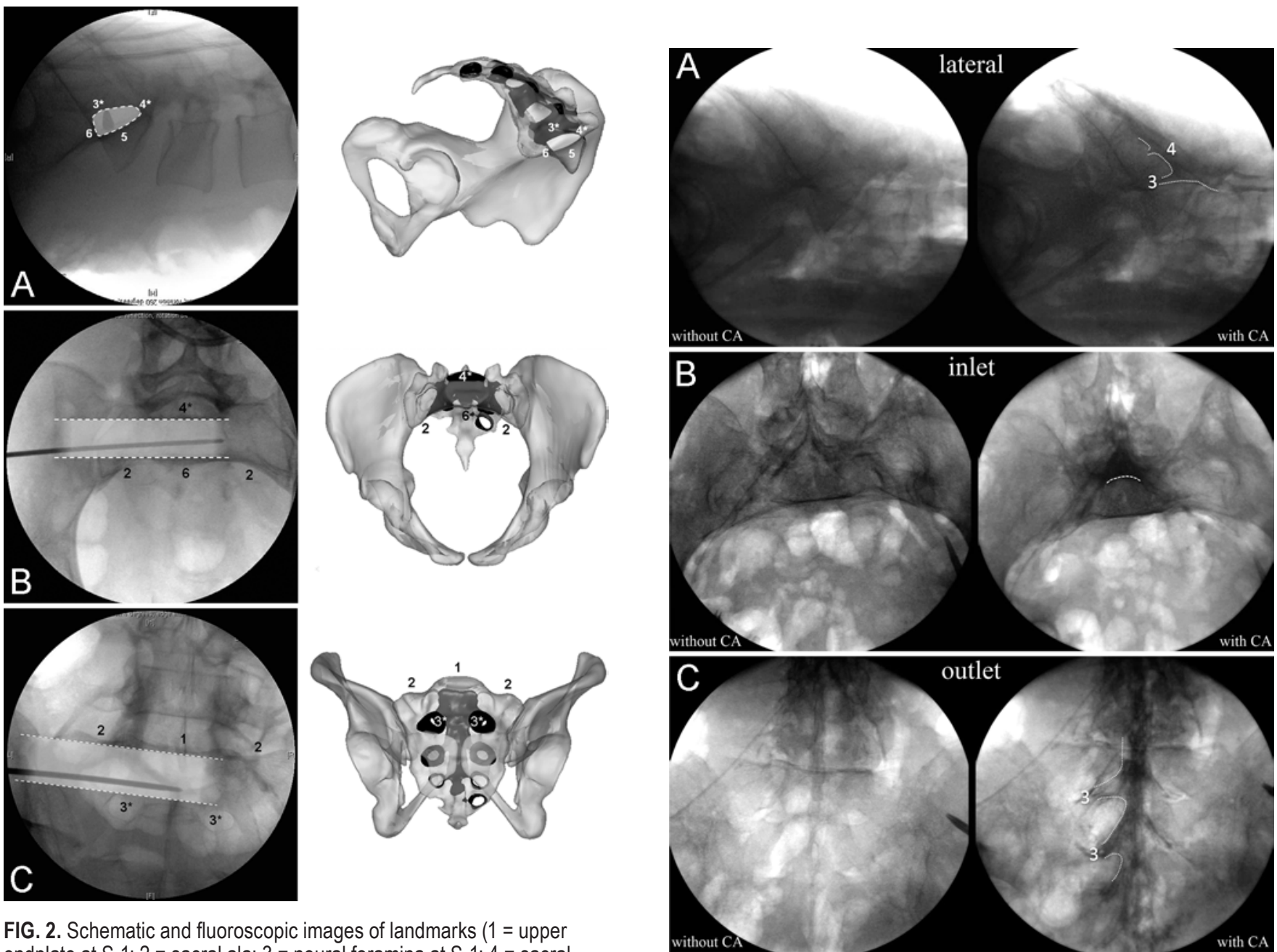

FIG 2. Schematic and fluoroscopic images of landmarks (1 = upper endplate at S-1; 2 = sacral ala; $3=$ neural foramina at S-1; $4=$ sacral canal; $5=$ linea terminalis; and $6=$ anterior wall at S-1) in different projections (lateral [A], inlet [B], and outlet [C]) of an individual (28 years of age) with good bone quality (the dashed lines mark the boundaries of the screw corridor, and the asterisks mark landmarks of the sacral cavity).

FIG. 3. Fluoroscopic images in standard projections (lateral [A], inlet [B], and outlet [C]) before and after CA injection. Locations of the individual landmarks in the sacral cavity are indicated $(3=$ neural foramina; $4=$ sacral canal; dashed lines represent bony margins of the innersacral cavity adjacent to the SI-screw corridor). 


\section{Results}

The score results for each specimen before and after CA injection are shown in Table 2. In almost all specimens, the native fluoroscopic views of the SI pelvic region provided inadequate visibility of landmarks, including the S-1 neural foramina and the sacral canal. This poor visibility was due to the apparent poor bone quality in the geriatric donors and also due to intestinal gas formed by the onset of autolysis. The mean visibility scores for the native views were 1.4 for the lateral projection, 2.4 for the inlet projection, and 2.1 for the outlet projection (the mean total visibility score was 5.9).

In all 8 specimens, the sacral hiatus could be easily punctured. The exploration of the sacral hiatus preceding the epidural injection of the CA took on average 5.3 minutes (range 2.1-8.2 minutes). Multiple attempts were not required. In one case, the needle placement resulted in an outflow of the CA via the venous sacral plexus and the superior gluteal vein to the common iliac vein. After gentle retraction of the needle, the CA was injected into the epidural space, and the assessment of the C-arm images was not affected by the residual CA in the pelvic venous system.

Injection of the CA increased visibility of the landmarks in all cases. The visibility score in the inlet and outlet views increased to 2.8 , while the score increased to 2.6 in the lateral projection (the mean total visibility score after CA injection was 8.1). In all projections, especially in the lateral view, the visibility of the landmarks was improved (Fig. 3). In specimens P-II and P-VI, the native images showed exceptional contrast in the bony structures, indicating relatively good bone quality, and the total summative score was already 7 and 8 , respectively, before the $\mathrm{CA}$ injection. Injection of the CA increased the visibility score to 8 and 9 in these 2 specimens, respectively. In each of the 8 human specimens, the epidural contrast enhancement increased the total score (Fig. 4 upper). On average, CA instillation increased visibility of the bony landmarks by $37 \%$.

For the intrasacral bony landmarks, such as the sacral canal itself and the neural foramina at S-1, the landmark score increased from an average of 1.5 (range 0-2) to 3 (range 2-4), with a maximum possible score of 4 . This represented a 2 -fold increase in information. In 4 speci- mens, identification of these landmarks was not possible without CA injection (Fig. 4 lower).

The statistical analysis of the effect of CA injection on the visibility of the individual landmarks in the 8 specimens and in all 3 standard views indicated that the epidural CA instillation significantly improved the visibility of the S-1 neural foramina and of the sacral canal (Table 3 ). However, the analysis detected a significant increase in the visibility scores only at the sacral canal in the lateral view ( $p<0.001)$ and at the S-1 neural foramina in the outlet view $(\mathrm{p}=0.03)$. Although the $\mathrm{CA}$ instillation distinctly increased visibility of the sacral canal in the inlet view and of the S-1 neural foramina in the lateral view, this increase was not statistically significant $(p=0.31$ and $p=0.47$, respectively).

\section{Discussion}

Transhiatal epidural injection is a widely used and standardized technique in anesthesia and pain management. The placement of a cannula under fluoroscopic guidance is the gold standard. ${ }^{12}$ The aim of this cadaveric study was to evaluate the feasibility of transhiatal epidural injection for enhancing visualization of bony landmarks during percutaneous SI screw placement. The detection of these landmarks after CA injection was compared with detection with a standard fluoroscopy method.

The risk of complications from a transhiatal epidural CA injection is quite low. Allergic reactions, thyroid dysfunction, and reduced renal filtration rates should be considered before the intraoperative use of a CA. An accidental intrathecal injection leads to a myelography-like result but is unproblematic because epidural contrasting can be easily achieved by retracting the needle a few millimeters..$^{12}$ However, the rising intrathecal pressure caused by the volume of the injected CA may lead to neurological symptoms, and the lower sacral roots may be injured iatrogenically. For these reasons, because the distance from the caudal end of the dural sac to the sacral hiatus has an average distance of $35-45 \mathrm{~mm}$, the needle should be advanced only for a short distance. ${ }^{12}$

In one of our specimens, outflow of the CA from the venous sacral plexus to the iliac vein was observed. After correcting the position of the spinal needle, the epidural

TABLE 2. Landmark visibility scores for each of the cadaveric specimens in the 3 fluoroscopic views and total summative score before and after epidural CA injection

\begin{tabular}{|c|c|c|c|c|c|c|c|c|}
\hline \multirow[b]{2}{*}{ Specimen } & \multicolumn{2}{|c|}{ Lat View } & \multicolumn{2}{|c|}{ Inlet View } & \multicolumn{2}{|c|}{ Outlet View } & \multicolumn{2}{|c|}{ Summative Score } \\
\hline & w/o CA & w/ CA & w/o CA & w/ CA & w/o CA & w/ CA & w/o CA & w/ CA \\
\hline P-I & 1 & 2 & 2 & 2 & 1 & 2 & 4 & 6 \\
\hline P-II & 2 & 3 & 3 & 3 & 3 & 3 & 8 & 9 \\
\hline P-III & 2 & 4 & 2 & 3 & 2 & 3 & 6 & 10 \\
\hline P-IV & 1 & 2 & 2 & 3 & 3 & 3 & 6 & 8 \\
\hline$P-V$ & 2 & 3 & 2 & 2 & 2 & 3 & 6 & 8 \\
\hline $\mathrm{P}-\mathrm{VI}$ & 1 & 2 & 3 & 3 & 3 & 3 & 7 & 8 \\
\hline P-VII & 1 & 3 & 3 & 3 & 2 & 3 & 6 & 9 \\
\hline P-VIII & 1 & 2 & 2 & 3 & 1 & 2 & 4 & 7 \\
\hline Mean score (range) & $1.4(1-2)$ & $2.6(2-4)$ & $2.4(2-3)$ & $2.8(2-3)$ & $2.1(1-3)$ & $2.8(2-3)$ & $5.9(4-8)$ & $8.1(6-10)$ \\
\hline
\end{tabular}



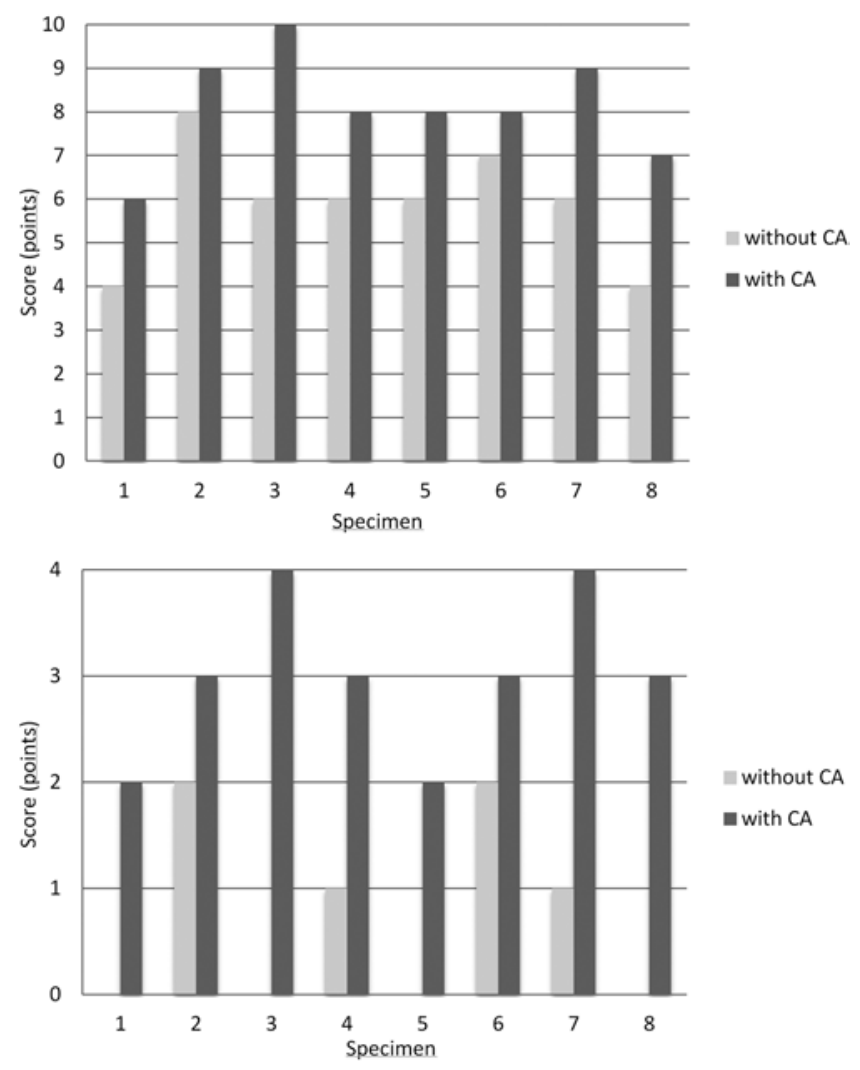

FIG. 4. Visibility scores before and after injection of the CA into the epidural space of 8 specimens. Total scores for all landmarks (upper) and scores for inner-sacral cavity landmarks, namely, the sacral channel and the S-1 neural foramina (lower).

space was properly contrasted, and a valid image assessment was possible. In clinical use, we expect that the venous circulation would dilute the CA much faster than in our cadaveric specimens.

According to the literature, a complete bony closure of the sacral hiatus is observed in $2 \%$ of the population. ${ }^{12} \mathrm{In}$ such cases, it is impossible to apply the transhiatal epidural CA injection method. This variation in bone structure can be easily identified on preoperative CT scans. Moreover, in approximately $6.3 \%$ of the population, the bony sacral canal fails to close. ${ }^{12}$ However, neither anatomical variation was found in our samples.

Limited visibility of essential bony landmarks can complicate secure SI screw placement, limiting or prohibiting the placement. Iatrogenic nerve lesions due to implant malposition have been reported to occur in up to $25 \%$ of cases during percutaneous fluoroscopic SI screw fixation. ${ }^{10,13,15}$ Recently, techniques for optimizing bony landmarks, such as intraoperative CT guidance, ${ }^{2}$ neuromonitoring, ${ }^{15}$ and the use of computer-assisted navigation systems, ${ }^{1,10}$ have been described, which have distinctly decreased screw malpositioning. However, these methods require more personnel and significantly more technical equipment. By comparison, the transhiatal application of $\mathrm{CA}$ is a straightforward and inexpensive method to enhance the visibility of essential bony landmarks during fluoroscopy, especially in osteoporotic bones. The total cost for a spinal cannulation and the CA is approximately $\$ 90$ for a single use.

In the specimens from geriatric individuals in our study, the low native fluoroscopic signal from pelvic structures was caused by poor bone quality. It reflects a common problem during percutaneous SI screw placement in older patients. The mean age at the time of death of our 8 donors was 78 years. Hence, this cohort represents a worst-case scenario for SI screw placement. We found that, in all of our specimens, epidural CA injection increased the information content of the C-arm images. When considering only the landmarks that can be contrast enhanced with CA injection, such as the sacral canal and the S-1 neural foramina, this enhancement was even more apparent. The corresponding scores were significantly higher, and in 4 cases, the landmarks became visible only after the CA injection.

Some limitations of this study should be mentioned. It remains unclear if epidural contrast enhancement of the intrasacral cavity system can be performed successfully in sacral fractures involving the neural foramina and the sacral canal (that is, in Denis Zones II and III). ${ }^{4}$ The CA outflow into adjacent soft tissues should be unproblematic for such patients; however, fluoroscopic visualization may be affected. In common transalar sacral fractures (Denis Zone I), observed as B2.1 injuries to the pelvic ring in older patients after a fall on the side, and in most insufficiency fractures, we do not expect impaired fluoroscopic visualization. However, in fracture types affecting the intrasacral cavity system, the CA may leak throughout the building deposits of the fracture line, and this leakage may affect fluoroscopic visualization. However, to exclude penetration of the CA into the inner-sacral cavities, we now routinely inject the CA during augmentation with polymethyl methacrylate of SI screws via the screw cannula directly into the preformed drill hole before cement aug-

TABLE 3. Crosstab analysis of the effect of transhiatal epidural CA injection on the visibility of the inner-sacral cavity landmarks in the 8 cadaveric specimens

\begin{tabular}{|c|c|c|c|c|c|c|c|c|}
\hline \multirow[b]{3}{*}{ Landmark } & \multicolumn{8}{|c|}{ No. of Specimens (\%) } \\
\hline & \multicolumn{2}{|c|}{ Sacral Canal Inlet View } & \multicolumn{2}{|c|}{ S-1 Neural Foramina Outlet View } & \multicolumn{2}{|c|}{ S-1 Neural Foramina Lat View } & \multicolumn{2}{|c|}{ Sacral Canal Lat View } \\
\hline & w/o CA & w/ CA & w/o CA & w/ CA & w/o CA & w/ CA & w/o CA & $\mathrm{CA}$ \\
\hline Visible & $2(25)$ & $5(62)$ & $0(0)$ & $5(62)$ & $6(75)$ & $8(100)$ & $0(0)$ & $8(100)$ \\
\hline Invisible & $6(75)$ & $3(38)$ & $8(100)$ & $3(38)$ & $2(25)$ & $0(0)$ & $8(100)$ & $0(0)$ \\
\hline Fisher exact test* & \multicolumn{2}{|c|}{$p=0.315$} & \multicolumn{2}{|c|}{$p=0.026$} & \multicolumn{2}{|c|}{$p=0.467$} & \multicolumn{2}{|c|}{$p=0.0002$} \\
\hline
\end{tabular}

* The $p$ values indicate the statistical signficance of differences in visibility of inner-sacral landmarks between the native view and the view after CA injection; differences were considered statistically significant at $p<0.05$. 
mentation. In our experience, most of the CA is evacuated within seconds by the high blood perfusion of the sacrum. Leakage of CA through the fracture line may be also of little concern because SI screw fixation is commonly performed some days after the initial trauma, and blood clots may inhibit any significant CA discharge.

Another limitation is that bone density was not measured in the specimens. However, it is widely accepted that the mineral content in bones from women 70 years or older and from men 80 years or older commonly leads to osteoporotic conditions, increasing the incidence of osteoporotic pelvic fractures..$^{5,8,9}$ An important limitation of the statistical analysis in the present study is the low number of specimens. Although the Fisher exact test permits accurate estimation of statistical significance from small samples, the results of the present study should be interpreted conservatively. Finally, this cadaveric study presents an initial feasibility trial of the presented method. The experimental setup focused on the enhanced visualization of bony landmarks by CA instillation into the epidural cavity of the inner sacrum. As such, we did not include the practical step of SI screw insertion. Further investigations are needed to evaluate the potential of our new method to improve patients' safety during SI screw fixation. Nonetheless, the results presented here are encouraging for the future use of transhiatal epidural CA injections in clinical applications.

\section{Conclusions}

The straightforward puncture of the sacral epidural space through the sacral hiatus is a viable and well-established method. The CA-mediated enhancement of the epidural space for visualization of the intrasacral bony landmarks improves the identification of these landmarks in $\mathrm{C}$-arm images. This improved identification significantly increases the safety of SI screw placement, especially in osteoporotic bone.

\section{References}

1. Arand M, Kinzl L, Gebhard F: Computer-guidance in percutaneous screw stabilization of the iliosacral joint. Clin Orthop Relat Res (422):201-207, 2004

2. Blake-Toker AM, Hawkins L, Nadalo L, Howard D, Arazoza A, Koonsman M, et al: CT guided percutaneous fixation of sacroiliac fractures in trauma patients. J Trauma 51:11171121,2001

3. Chen CP, Tang SF, Hsu TC, Tsai WC, Liu HP, Chen MJ, et al: Ultrasound guidance in caudal epidural needle placement. Anesthesiology 101:181-184, 2004

4. Denis F, Davis S, Comfort T: Sacral fractures: an important problem. Retrospective analysis of 236 cases. Clin Orthop Relat Res 227:67-81, 1988
5. Link TM: Osteoporosis imaging: state of the art and advanced imaging. Radiology 263:3-17, 2012

6. Mendel T, Appelt K, Kuhn P, Suhm N: [Bony sacroiliac corridor. A virtual volume model for the accurate insertion of transarticular screws.] Unfallchirurg 111:19-26, 2008 (Ger)

7. Mendel T, Noser H, Wohlrab D, Stock K, Radetzki F: The lateral sacral triangle-a decision support for secure transverse sacroiliac screw insertion. Injury 42:1164-1170, 2011

8. Nanninga GL, de Leur K, Panneman MJ, van der Elst M, Hartholt KA: Increasing rates of pelvic fractures among older adults: The Netherlands, 1986-2011. Age Ageing 43:648-653, 2014

9. Parkkari J, Kannus P, Niemi S, Pasanen M, Järvinen M, Lüthje P, et al: Secular trends in osteoporotic pelvic fractures in Finland: number and incidence of fractures in 1970-1991 and prediction for the future. Calcif Tissue Int 59:79-83, 1996

10. Schep NW, Haverlag R, van Vugt AB: Computer-assisted versus conventional surgery for insertion of 96 cannulated iliosacral screws in patients with postpartum pelvic pain. J Trauma 57:1299-1302, 2004

11. Senoglu N, Senoglu M, Oksuz H, Gumusalan Y, Yuksel KZ, Zencirci B, et al: Landmarks of the sacral hiatus for caudal epidural block: an anatomical study. Br J Anaesth 95:692695, 2005

12. Stitz MY, Sommer HM: Accuracy of blind versus fluoroscopically guided caudal epidural injection. Spine (Phila Pa 1976) 24:1371-1376, 1999

13. Tonetti J, Cazal C, Eid A, Badulescu A, Martinez T, Vouaillat $\mathrm{H}$, et al: [Neurological damage in pelvic injuries: a continuous prospective series of 50 pelvic injuries treated with an iliosacral lag screw.] Rev Chir Orthop Reparatrice Appar Mot 90:122-131, $2004(\mathrm{Fr})$

14. Tsui BC, Tarkkila P, Gupta S, Kearney R: Confirmation of caudal needle placement using nerve stimulation. Anesthesiology 91:374-378, 1999

15. Webb LX, de Araujo W, Donofrio P, Santos C, Walker FO, Olympio MA, et al: Electromyography monitoring for percutaneous placement of iliosacral screws. J Orthop Trauma 14:245-254, 2000

16. Weinstein SM, Herring SA, Derby R: Contemporary concepts in spine care. Epidural steroid injections. Spine (Phila Pa 1976) 20:1842-1846, 1995

\section{Author Contributions}

Conception and design: Mendel, Hofmann, Goehre. Acquisition of data: Mendel, Radetzki. Analysis and interpretation of data: Mendel, Goehre. Critically revising the article: Schwan. Reviewed submitted version of manuscript: Mendel. Statistical analysis: Schwan. Study supervision: Hofmann.

\section{Correspondence}

Thomas Mendel, Department of Trauma Surgery, BG-Kliniken Bergmannstrost, Merseburger Str. 165, 06112 Halle (Saale), Germany. email: thomasmendel@hotmail.com. 